\title{
In vivo dynamic monitoring of the biological behavior of labeled C6 glioma by MRI
}

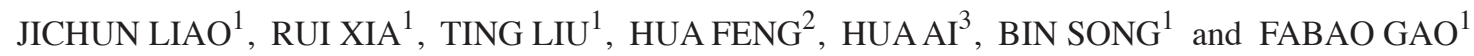 \\ ${ }^{1}$ Department of Radiology, West China Hospital, Sichuan University, Chengdu, Sichuan 610041; \\ ${ }^{2}$ Department of Neurosurgery, Southwest Hospital, Third Military Medical University, Chongqing 400038; \\ ${ }^{3}$ National Engineering Research Center for Biomaterials, Sichuan University, Chengdu, Sichuan 610065, P.R. China
}

Received November 2, 2012; Accepted February 21, 2013

DOI: $10.3892 / \mathrm{mmr} .2013 .1369$

\begin{abstract}
Gliomas are the most common type of intracranial tumor and have the highest rate of mortality. The aims of this study were to investigate the long-term course and biological behavior of orthotopically implanted C6 gliomas and to dynamically monitor the distribution of superparamagnetic iron oxide (SPIO) nanocomposite-labeled C6 glioma cells in rats using 7.0T MRI. We observed that in the MRI of the rats implanted with SPIO-labeled cells, there were pronounced hypointense signal bands, which faded over time, but remained visible up to day 27 after implantation. We observed that the first tumors were detected as early as 2 days after implantation, presenting as slightly hyperintense regions with indefinite boundaries in the T1-weighted images (T1WIs). On the 9th day, thick tumor feeder vessels, $\sim 0.2 \mathrm{~mm}$ in diameter, were observed and these increased rapidly over time. Edema was observed in the labeled and unlabeled groups in the T2WIs. Both the central hypointense signal area and the peripheral cogwheel-shaped hypointense signal band in the tumor were observed on the post-contrast T1WIs, in accordance with the necrosis observed in the photomicrographs following hematoxylin and eosin (HE) staining. In conclusion, labeling tumor cells with SPIO and performing an MRI scan dynamically monitors the development and biological behavior of glioma at a very early stage.
\end{abstract}

\section{Introduction}

Gliomas are the most common type of intracranial tumor and have the highest rate of mortality (1). Despite advanced diagnostics and treatments, the average survival time does not exceed 15 months. Matsukado et al observed that more than half of untreated gliomas reached the contralateral

Correspondence to: Dr Fabao Gao, Department of Radiology, West China Hospital, Sichuan University, 37 Guoxuexiang Road, Chengdu, Sichuan 610041, P.R. China

E-mail: gaofabao@yahoo.com

Key words: MRI, glioma, rat, superparamagnetic iron oxide hemisphere (2).Malignant gliomas display a prominent degree of invasiveness into the surrounding normal brain tissue (3), which compromises therapeutic efforts and ultimately explains the high rates of recurrence and the poor prognosis for patients. The implantation of a C6 glioma in the rat brain and subsequent study of its biological behavior, including the extent of tumor development, spontaneous regression of the glioma, the best experimental time window, cell invasion pathological characteristics and neoangiogenesis, have been beneficial to the clinical treatment of gliomas.

A number of in vivo imaging modalities are used to assess tumor development in preclinical animal models. MRI is considered to be the method of choice for studying the biological behavior of a tumor, as it enables the imaging of an entire organ or animal longitudinally over time. MRI has the best soft-tissue contrast, excellent sensitivity and a spatial resolution approaching the cellular level with a voxel size of $10 \mu \mathrm{m}$ in vitro and $50 \mu \mathrm{m}$ in vivo. Furthermore, MRI provides information regarding tumor location and size, the extent of edema, relative blood volume fraction and blood-brain barrier status without ionizing radiation (4). In addition, MRI is thought to be the most promising technique for non-invasive cell tracking in vivo. MRI negative contrast agents, such as superparamagnetic iron oxide (SPIO) (5) and micron-sized iron oxide particles $(6,7)$, are the most frequently used, and have been used to magnetically label cells ex vivo, providing researchers with the ability to monitor the distribution and migration of these cells in animals and humans in vivo (8-10). Intracellular SPIOs placed in a magnetic field cause signal dephasing due to $\mathrm{B}_{0}$ inhomogeneities induced close to the cells. The disruption of the magnetic field extends to a much larger distance than the actual size of the SPIO nanoparticles, making it possible to detect low numbers of cells, even single cells (11), in vivo.

In designing this study, we had two objectives: i) to investigate the long-term course and biological behavior of orthotopically implanted C6 gliomas; and ii) to dynamically monitor the distribution of SPIO-labeled C6 cells in rats with MRI.

\section{Materials and methods}

Cell culture and labeling. Rat C6 glioma cell lines were provided by the State Key Laboratory of Biotherapy (Sichuan, 
China) and cultured at $37^{\circ} \mathrm{C}$ in a humidified atmosphere with $5 \% \mathrm{CO}_{2}$ in Dulbecco's modified Eagle's medium (DMEM; Sigma, St. Louis, MO, USA) supplemented with $10 \%$ heat-inactivated foetal bovine serum, $100 \mathrm{kU} / 1$ penicillin and $100 \mathrm{mg} / \mathrm{l}$ streptomycin. Superparamagnetic nanocrystals for labeling were amphiphilic polyethyleneimine (PEI)/SPIO nanocomposites (mean diameter $<100 \mathrm{~nm}$ ), provided by the National Engineering Research Center for Biomaterials, Sichuan University. The SPIO nanoparticles were added to DMEM (5 $\mu \mathrm{g} \mathrm{Fe} / \mathrm{ml})$ (12). The C6 cells were incubated in the culture medium for $12 \mathrm{~h}$ at $37^{\circ} \mathrm{C}$ in a $5 \% \mathrm{CO}_{2}$ atmosphere. The cells were washed thoroughly with phosphate-buffered saline (PBS) to remove unincorporated SPIOs and collected. In the control group, C6 cells were cultured without SPIOs.

Trypan blue viability assay. Labeled and unlabeled cells were suspended in PBS at a concentration of $1 \times 10^{6} / \mathrm{ml}$ and mixed with $0.4 \%$ trypan blue dye in a $1: 1$ ratio. The mixture $(10 \mu \mathrm{l})$ was loaded onto a hemocytometer and the cells were counted. Cells with an intact membrane excluded the dye and were considered to be live cells. The percentages of live and dead cells were determined.

Animal model. The local Institutional Animal Care and Use Committee approved all animal procedures. The hosts were 12 male Wistar rats weighing 300-350 g, obtained from the West China Experimental Animal Center. They were kept on a regular daylight schedule with rodent animal chow and water ad libitum. For implantation surgery, each animal was anesthetized with $10 \%$ chloral hydrate $(4 \mathrm{ml} / \mathrm{kg}$ i.p. $)$ prior to surgery. The head was placed on a stereotactic head holder and a scalp incision was made along the median line. A burr hole $1 \mathrm{~mm}$ in diameter was drilled into the skull, $3 \mathrm{~mm}$ lateral to the bregma. A $25 \mu \mathrm{l}$ cell suspension ( $1 \times 10^{6}$ C6 cells in DMEM-free serum) was injected into the right caudate nucleus, at a depth of $6 \mathrm{~mm}$ beneath the skull, using a Hamilton syringe. The needle was slowly removed 5 min after injection and the burr hole was plugged with vegetal wax.

MRI. All experiments were performed under anesthesia with the following parameters: $5 \%$ isoflurane for induction, and $2 \%$ isoflurane for maintenance in $60 \%$ air and $40 \%$ oxygen. The rectal temperature was maintained at $37.0 \pm 0.5^{\circ} \mathrm{C}$ throughout the experiments using a warming pad with hot water circulation, which was placed on the back of the animal. All measurements were performed on a Bruker Biospec 7T/30 cm horizontal bore magnet (Bruker BioSpin MRI, Ettlingen, Germany) using a volume coil for the rat head (inner diameter, $40 \mathrm{~mm}$; outer diameter, $75 \mathrm{~mm}$; length, $100 \mathrm{~mm}$ ) at multiple time-points (Table I). The parameters were as follows: matrix (MTX), 256x256; FOV, $3 \mathrm{~cm}$; slice thickness, $1 \mathrm{~mm}$; interslice, $1 \mathrm{~mm}$. MSME T1 TR/TE, 561/14 msec; FA, $180^{\circ}$; NEX, 4; total scanning time, $9 \mathrm{~min}, 34 \mathrm{sec}, 502 \mathrm{msec}$; RARE T2 TR/TE, 3000/45 msec; FA, $180^{\circ}$; NEX, 4; total scanning time, $6 \mathrm{~min}, 24 \mathrm{sec}, 0 \mathrm{msec}$. Post-contrast T1-weighted images (T1WIs) were obtained $5 \mathrm{~min}$ after the intravenous injection of $0.1 \mathrm{mmol} / \mathrm{kg}$ gadopentetate dimeglumine contrast agent (Bayer Schering Pharma AG, Berlin, Germany).
Table I. Scanning time points of every rat in the experimental group and control group.

\begin{tabular}{|c|c|c|c|c|c|}
\hline \multirow{2}{*}{$\begin{array}{l}\text { Rat } \\
\text { No. } 1\end{array}$} & \multicolumn{5}{|c|}{ Scanning time-point } \\
\hline & $2 \mathrm{~h}$ & 1 day & 11 days & 20 days & \\
\hline No. 2 & 1 day & 3 days & 9 days & 20 days & \\
\hline No. 3 & 1 day & 2 days & 3 days & 7 days & \\
\hline No. 4 & $2 \mathrm{~h}$ & 2 days & 3 days & 9 days & 27 days \\
\hline No. 5 & 2 days & 7 days & & & \\
\hline No. 6 & 2 days & 11 days & 20 days & 27 days & \\
\hline No. 7 & 11 days & 20 days & & & \\
\hline No. 8 & 2 days & 3 days & 9 days & 20 days & 27 days \\
\hline
\end{tabular}

$\mathrm{B}$, Control group

\begin{tabular}{llcl}
\hline Rat & \multicolumn{3}{c}{ Scanning time-point } \\
\hline No. 1 & 10 days & 20 days & 27 days \\
No. 2 & 10 days & 20 days & \\
No. 3 & 10 days & 20 days & 27 days \\
No. 4 & 10 days & 20 days & \\
\hline
\end{tabular}

Histological examination. All animals were sacrificed after MRI study for histological examination. Brains were immersed in $4 \%$ paraformaldehyde and embedded in paraffin; $10 \mu \mathrm{m}$ coronal sections were then cut through the cortical hemispheres and stained with hematoxylin and eosin (HE) and Perl's solution, the latter of which was used for iron detection.

Data analysis. All images were processed using ParaVision software (v.5.0; Bruker Biospin MRI). Regions of interest (ROIs) were drawn manually at four regions in the tumor on the biggest slice of the tumors for each model. In each case, contralateral ROIs of equivalent surface area were also drawn. Data are presented as the means \pm SD. Statistically significant differences between the tumors and the contralateral areas and between the labeled experimental and unlabeled control groups were analyzed by an unpaired, two-tailed Student's t-test using statistical software (SPSS 13.0; SPSS, Inc., Chicago, IL, USA). P<0.05 was considered to indicate a statistically significant difference.

\section{Results}

The viabilities of the labeled and unlabeled cells were analyzed using a trypan blue assay. We observed no significant difference between the viabilities of the labeled $(93.5 \%)$ and unlabeled cells $(94.2 \%)$.

All 12 rats tolerated infusion of the SPIO-labeled and unlabeled C6 cells and no difference in clinical course was observed between the animals with implanted tumors who received labeled cells and the controls who received unlabeled cells. The C6 cells did not form a tumor in one rat; therefore, 

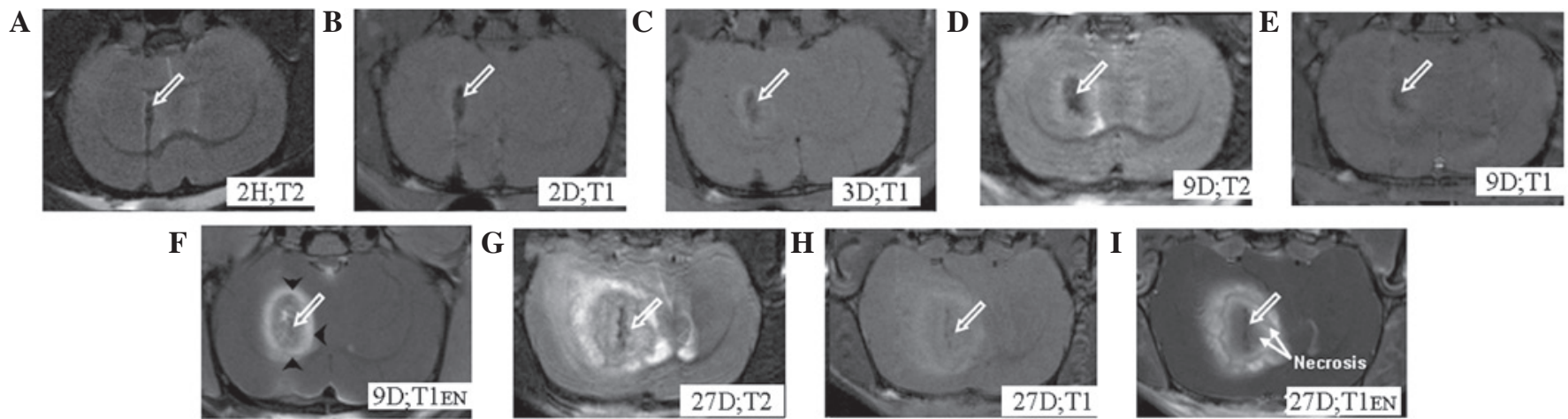

Figure 1. Experimental group. Serial MR images at different time-points after superparamagnetic iron oxide (SPIO)-labeled C6 glioma cells were implanted in model rat brains. A pronounced hypointense signal band is observed that fades gradually over time (hollow arrow). Tumors are significantly enhanced (arrow head), with the exception of necrotic areas (arrow). H, hours; D, days; EN, enhancement.
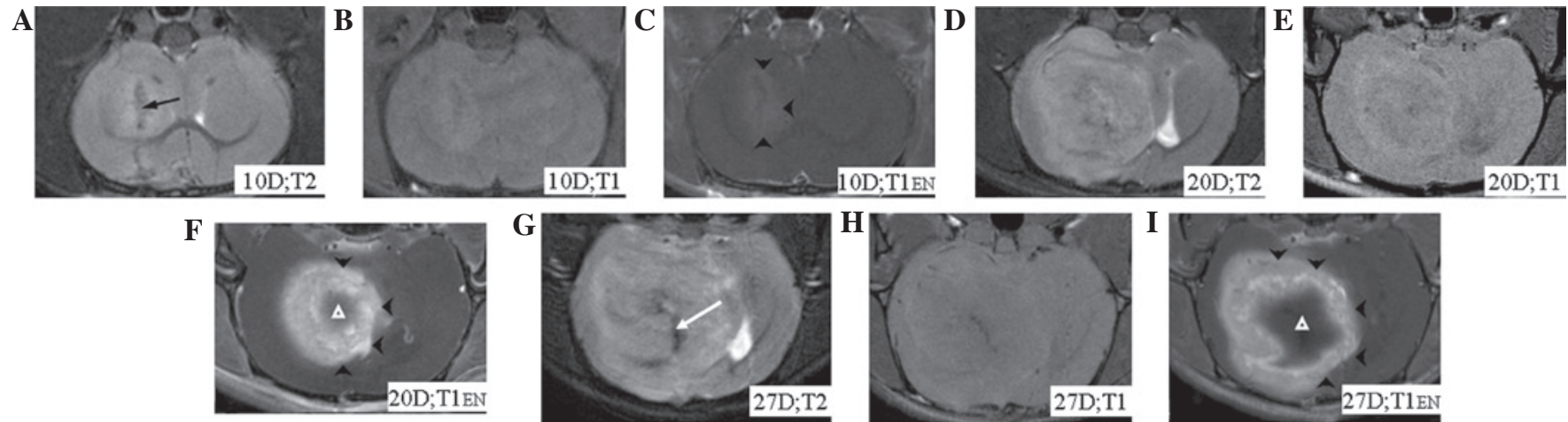

Figure 2. Control group. Serial MR images at various time-points after implantation of unlabeled C6 glioma cells. No hypointense signal bands were observed. Tumors were enhanced (arrow head), with the exception of necrotic areas (white arrow head). Black arrow, needle tract; white arrow, vascularization; D, days; EN, enhancement.
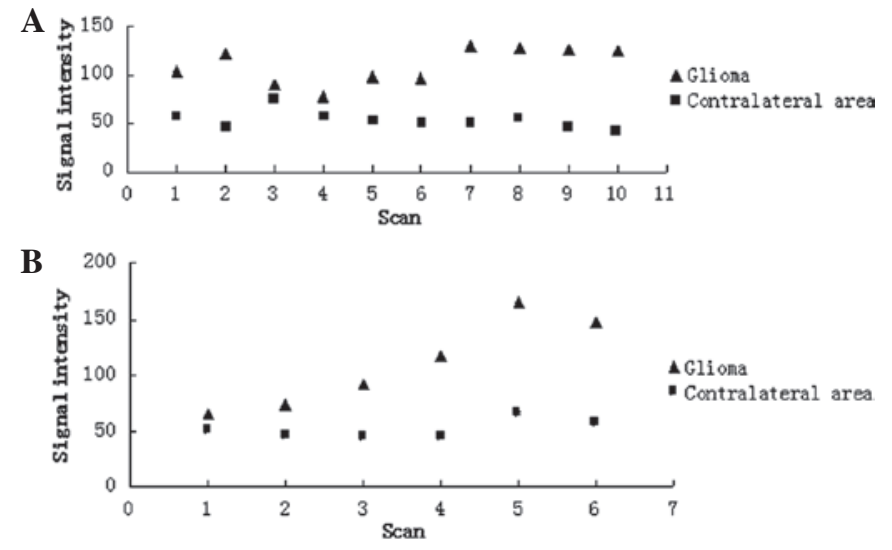

Figure 3. T2 signal intensity of the gliomas and contralateral areas in (A) labeled experimental and (B) unlabeled control animals (scan indicates each MR examination including post-contrast T1 images, which may be of different animals or examination of the same animal at a different time-point).

the tumor formation rate was $92 \%$. In the experimental group, we observed pronounced hypointense signal bands, which gradually faded over time, but remained visible on the MRI 27 days after implantation (Fig. 1). All animals were euthanized after day 27. In the control group, no hypointense signal band was observed (Fig. 2). MRI images from the experimental group revealed tumors from the 2 nd day after implantation; these presented as slightly hyperintense regions with indefinite boundaries surrounding focal hypointensities on the T1WIs. The tumors were enhanced on the post-contrast T1WIs, with the exception of the necrotic areas (Fig. 1). Following the injection of contrast media, the signal intensity was found to increase $(\mathrm{P}<0.05)$ in the tumor regions of the labeled experimental and unlabeled control group animals when compared with the contralateral tissue values of the same animals. There was no statistically significant difference $(\mathrm{P}>0.05)$ in the signal intensity of the tumor regions between the labeled experimental and unlabeled control groups (Fig. 3).

On the 9th day after implantation, thick tumor feeder vessels $\sim 0.2 \mathrm{~mm}$ in diameter were observed and these increased rapidly over time. On day 27 , diffuse, thick tumor feeder vessels were observed around as well as within the tumors (Fig. 4). Edema was observed in both groups, and presented as hyperintense regions around the tumors on the T2WIs. Besides the central areas of the tumors (around the pronounced hypointense signal bands), cogwheel-shaped bands were observed surrounding these central areas and neither were enhanced in the post-contrast T1WIs (Fig. 5A) in accordance with the necrosis observed in the photomicrographs following HE staining (Fig. 5B). The layers of necrosis were so clear in the MRI that these images were very similar to the photomicrographs of the HE-stained specimens. The blue dots in the Perl's-stained photomicrograph represent nanoparticles in the tumor cells (Fig. 5A, insert). No blue dots were observed in the control group. 
A

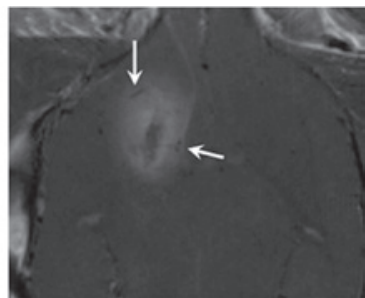

B

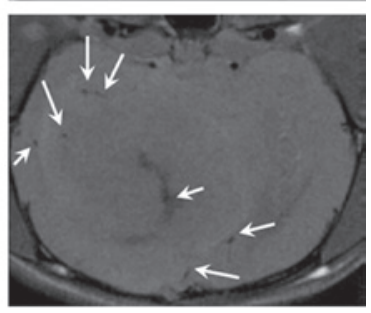

Figure 4. Arrows indicate the thick tumor feeder vessels (A) 9 days and (B) 27 days after the implantation of $\mathrm{C} 6$ cells.

In the experimental group, hypointense regions were observed not only in the right caudate nucleus but also in the right lateral ventricle in 4/8 model animals; these were caused by some of the labeled tumor cells flowing into the lateral ventricle. In later MRI images, the tumor cells in the right caudate nucleus, as well as those in the lateral ventricle, had grown into tumors. The implanted tumor was generally irregular (Fig. 6), but in the control group it was not possible to discern whether the implanted tumor cells were only in the right caudate nucleus or if they had flowed into the lateral ventricle in the early stages, as the signal from the unlabeled cells was similar to that from normal brain tissue.

\section{Discussion}

C6 cells have been implanted in Sprague-Dawley, BDX, BDIX and Wistar rat strains for glioma modeling. It is currently assumed that C6 cells were first produced in Wistar rats exposed to N-nitrosomethylurea (13). The C6 glioma model is simple, reliable and easily reproducible; it also has a high tumor formation rate. In this study, tumors were formed in $92 \%(11 / 12)$ of the model animals. A study by Vince et al (14) confirmed that the C6 glioma model best mimics the cellular biological behavior of early glioma progression in humans; C6 cells are very similar to human glioma cells in terms of the gene expression involved in tumor progression (15). The present study monitored the tumors from $1 \mathrm{~h}$ to 27 days after implantation using 7.0T MRI and it was discovered that it was possible to detect tumors as early as 2 days after implantation. By contrast, Doblas et al recently reported a latency time in C6 gliomas of $11 \pm 1.93$ days (16). However, they did not take MRI images of the rats until 7 days after implantation, whereas we took images as early as $1 \mathrm{~h}$ after implantation to enable monitoring of the early biological behavior of C6 gliomas. To the best of our knowledge, this is the first report demonstrating that C6 gliomas may be detected as early as 2 days after implantation.

MRI provides a more rapid and complete account of the cell and tumor burden than is possible by histological analysis, and permits repeated sampling of individual experimental animals over long periods of time. In our study, the central
A

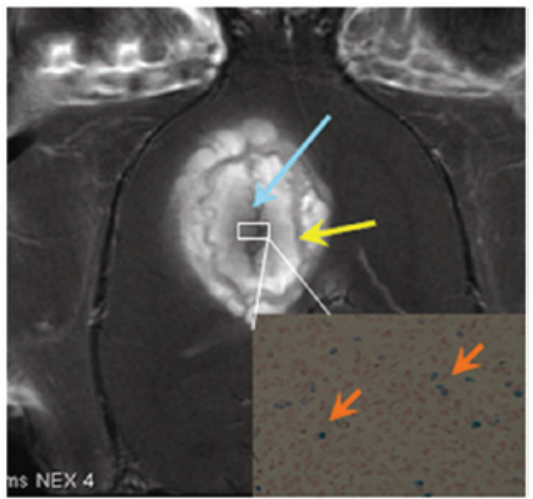

$\mathbf{B}$

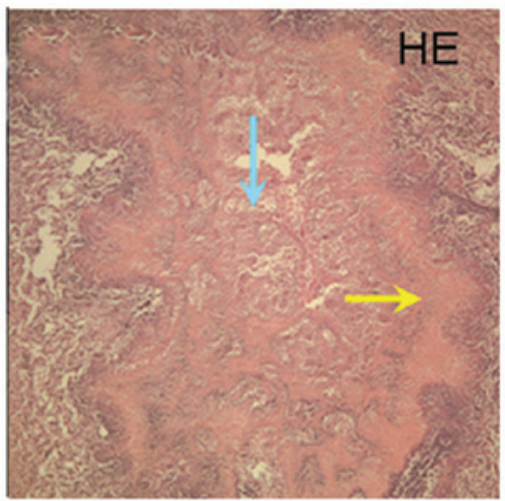

Figure 5. Post-contrast T1WIs (A) in accordance with the photomicrograph of the hematoxylin and eosin (HE)-stained specimen (magnification, $\mathrm{x} 4$ ). (B) Central necrosis (blue arrow) surrounded by a cogwheel shaped necrosis (yellow arrow). Blue dots on Perl's-stained specimens (magnification, x40) represent nanoparticles in tumor cells (red arrow). T1WI, T1-weighted image.

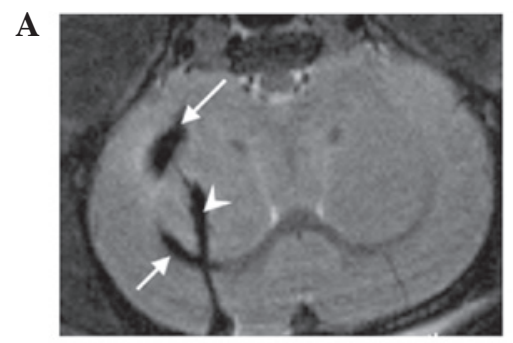

B

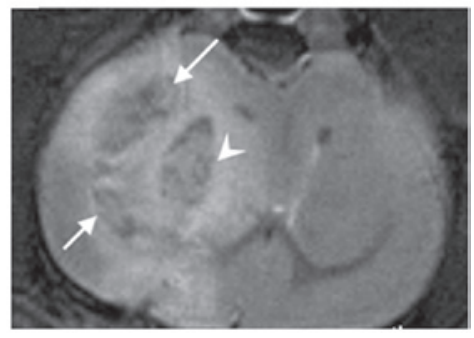

Figure 6. T2WI images of a rat head (A) 2 days and (B) 20 days after implantation of labeled C6 cells. Arrow heads indicate labeled C6 cells in the right caudate nucleus and arrows indicate cells in the lateral ventricle. T2WI, T2-weighted image.

hypointense signal area and the peripheral cogwheel-shaped hypointense signal band in the tumor were observed on the post-contrast T1WIs, in accordance with the necrosis observed in the photomicrographs of HE-stained specimens and the layers of necrosis were so clear that the MRI images were very similar to the photomicrographs of the HE-stained specimens. 
There has been no other report of these manifestations of the C6 glioma model using MRI. With MRI, we were able to image entire brain volumes in vivo with scan times $<10 \mathrm{~min} / \mathrm{animal}$, in contrast to the many hours required for histological assessment of cell and tumor distribution of just a portion of a rat brain at a single time-point. Labeling tumor cells with SPIO and performing an MRI scan dynamically monitors the development and biological behavior of glioma at a very early stage.

SPIOs have been used as ex vivo cell labeling agents for various types of mammalian cells and for in vivo cellular MRI (17), including in tumor cells (5), dendritic cells (18), stem cells (19) and microglia (20), and have recently been used in a clinical environment (18). Techniques for labeling cells have been extensively studied. As previously reported, SPIO labeling procedures have been established that do not affect cell viability, proliferation or differentiation potential in vitro $(21,22)$. However, Schäfer et al $(23)$ revealed that SPIO or ultrasmall SPIO (USPIO) labeling without a transfection reagent has a biological impact on mesenchymal stem cells (MSCs) by upregulating the transferrin receptor and the authors suggest caution when using this labeling procedure in clinical applications. Furthermore, SPIO labeling with a transfection reagent coats the cellular surface. In this study, we labeled tumor cells without a transfection reagent. We used self-assembly amphiphilic PEI/SPIO nanocomposites, which label cells at low dosages, have good biocompatibility and high labeling rates and no detrimental effects on cell proliferation (12). It remains unknown as to whether the transferrin receptor in tumor cells is upregulated as it is in the MSCs. Further experiments are required to elucidate the mechanisms and consequences of these effects.

We labeled C6 cells with SPIOs, ensuring that they appeared hypointense on T2WIs, enabling easy differentiation of these cells from normal brain tissue and thus also the localization of the implanted cells. In 4/8 experimental animals, the labeled tumor cells flowed into the lateral ventricle and later grew into particularly irregular tumors. Thus, labeling cells with SPIOs prior to implantation facilitates early MRI scanning and detection of the localization of implanted cells, allowing confirmation that the cells are in the expected location and the ability to remove unfavourable models at an early stage.

In the experimental group, we observed pronounced hypointense signal bands that faded over time but remained visible on day 27 after implantation, our study endpoint, indicating that the SPIO-labeled cells create hypointense signals that last for at least 27 days. One of the drawbacks of labeling cells with exogenous nanoparticles is the loss of signal over time. This loss may be attributed to either biodegradation or dilution of the contrast agent in asymmetric cell division (24). Arbab et al observed that the ability to detect Feridex-labeled cells with Perl's stain was lost after 5-8 cell divisions (22). One approach for overcoming such limitations is to use MRI reporter genes where the daughter cells retain a constant amount of the contrast-enhancing agent after each cell division $(25,26)$, however, these labels are less sensitive than SPIOs and the procedure is more complicated. In many cases, it is difficult to distinguish labeled cells from other hypointense regions in T2/T2*-weighted MRI images. Hypointensities may have a physiological origin, such as hemoglobin in blood, a pathological origin, such as blood clots, or an experimental origin from traumatic procedures, such as cell injections (Fig. 2A). Hypointensities on MRI images remain a major obstacle in the attempt to increase the specificity of cell tracking, which prevents this method from being used in certain applications, particularly those that involve trauma and hemorrhage. Using positive contrast media solves this problem, however, the sensibility of positive contrast media is not as high as that of negative contrast media.

\section{Acknowledgements}

This study was supported by a grant from the Major State Basic Research Development of China (973 Program, No. 2011CB935800) and the National Natural Science Foundation of China (No. 81071204). The authors are grateful to the State Key Laboratory of Biotherapy for providing the $\mathrm{C} 6$ glioma cell line.

\section{References}

1. Wrensch M, Minn Y, Chew T, Bondy M and Berger MS: Epidemiology of primary brain tumors: current concepts and review of the literature. Neuro Oncol 4: 278-299, 2002.

2. Matsukado Y, Maccarty CS and Kernohan JW: The growth of glioblastoma multiforme (astrocytomas, grades 3 and 4) in neurosurgical practice. J Neurosurg 18: 636-644, 1961.

3. Demuth T and Berens ME: Molecular mechanisms of glioma cell migration and invasion. J Neurooncol 70: 217-228, 2004.

4. Weissleder R: Scaling down imaging: molecular mapping of cancer in mice. Nat Rev Cancer 2: 11-18, 2002.

5. Zhang F, Xie J, Liu G, He Y, Lu G and Chen X: In vivo MRI tracking of cell invasion and migration in a rat glioma model. Mol Imaging Biol 13: 695-701, 2011.

6. Shapiro EM, Skrtic S, Sharer K, Hill JM, Dunbar CE and Koretsky AP: MRI detection of single particles for cellular imaging. Proc Natl Acad Sci USA 101: 10901-10906, 2004.

7. Walton RM, Magnitsky SG, Seiler GS, Poptani H and Wolfe JH: Transplantation and magnetic resonance imaging of canine neural progenitor cell grafts in the postnatal dog brain. J Neuropathol Exp Neurol 67: 954-962, 2008.

8. Bulte JW and Kraitchman DL: Iron oxide MR contrast agents for molecular and cellular imaging. NMR Biomed 17: 484-499, 2004.

9. Valable S, Barbier EL, Bernaudin M, Roussel S, Segebarth C, Petit E and Rémy C: In vivo MRI tracking of exogenous monocytes/macrophages targeting brain tumors in a rat model of glioma. Neuroimage 40: 973-983, 2008.

10. Reddy AM, Kwak BK, Shim HJ, Ahn C, Lee HS, Suh YJ and Park ES: In vivo tracking of mesenchymal stem cells labeled with a novel chitosan-coated superparamagnetic iron oxide nanoparticles using 3.0T MRI. J Korean Med Sci 25: 211-219, 2010.

11. Heyn C, Ronald JA, Ramadan SS, et al: In vivo MRI of cancer cell fate at the single-cell level in a mouse model of breast cancer metastasis to the brain. Magn Reson Med 56: 1001-1010, 2006.

12. Liu G, Xia C, Wang Z, Lv F, Gao F, Gong Q, Song B, Ai H and $\mathrm{Gu} \mathrm{Z}$ : Magnetic resonance imaging probes for labeling of chondrocyte cells. J Mater Sci Mater Med 22: 601-606, 2011.

13. Benda P, Lightbody J, Sato G, Levine L and Sweet W: Differentiated rat glial cell strain in tissue culture. Science 161: 370-371, 1968.

14. Vince GH, Bendszus M, Schweitzer T, Goldbrunner RH, Hildebrandt S, Tilgner J, Klein R, Solymosi L, Christian Tonn J and Roosen K: Spontaneous regression of experimental gliomas--an immunohistochemical and MRI study of the C6 glioma spheroid implantation model. Exp Neurol 190: 478-485, 2004.

15. Sibenaller ZA, Etame AB, Ali MM, Barua M, Braun TA, Casavant TL and Ryken TC: Genetic characterization of commonly used glioma cell lines in the rat animal model system. Neurosurg Focus 19: E1, 2005.

16. Doblas S, He T, Saunders D, Pearson J, Hoyle J, Smith N, Lerner M and Towner RA: Glioma morphology and tumor-induced vascular alterations revealed in seven rodent glioma models by in vivo magnetic resonance imaging and angiography. J Magn Reson Imaging 32: 267-275, 2010. 
17. Corot C, Robert P, Idée JM and Port M: Recent advances in iron oxide nanocrystal technology for medical imaging. Adv Drug Deliv Rev 58: 1471-1504, 2006.

18. de Vries IJ, Lesterhuis WJ, Barentsz JO, et al: Magnetic resonance tracking of dendritic cells in melanoma patients for monitoring of cellular therapy. Nat Biotechnol 23: 1407-1413, 2005.

19. Rice HE, Hsu EW, Sheng H, Evenson DA, Freemerman AJ, et al: Superparamagnetic iron oxide labeling and transplantation of adipose-derived stem cells in middle cerebral artery occlusion-injured mice. AJR Am J Roentgenol 188: 1101-1108, 2007.

20. Fleige G, Nolte C, Synowitz M, Seeberger F, Kettenmann H and Zimmer C: Magnetic labeling of activated microglia in experimental gliomas. Neoplasia 3: 489-499, 2001.

21. Meng XX, Wan JQ, Jing M, Zhao SG, Cai W and Liu EZ: Specific targeting of gliomas with multifunctional superparamagnetic iron oxide nanoparticle optical and magnetic resonance imaging contrast agents. Acta Pharmacol Sin 28: 2019-2026, 2007.

22. Arbab AS, Bashaw LA, Miller BR, Jordan EK, Lewis BK, Kalish $\mathrm{H}$ and Frank JA: Characterization of biophysical and metabolic properties of cells labeled with superparamagnetic iron oxide nanoparticles and transfection agent for cellular MR imaging. Radiology 229: 838-846, 2003.
23. Schäfer R, Kehlbach R, Wiskirchen J, Bantleon R, Pintaske J, Brehm BR, Gerber A, Wolburg H, Claussen CD and Northoff H: Transferrin receptor upregulation: in vitro labeling of rat mesenchymal stem cells with superparamagnetic iron oxide. Radiology 244: 514-523, 2007.

24. Walczak P, Kedziorek DA, Gilad AA, Barnett BP and Bulte JW: Applicability and limitations of MR tracking of neural stem cells with asymmetric cell division and rapid turnover: the case of the shiverer dysmyelinated mouse brain. Magn Reson Med 58: 261-269, 2007.

25. Gilad AA, Winnard PT Jr, van Zijl PC and Bulte JW: Developing MR reporter genes: promises and pitfalls. NMR Biomed 20: 275-290, 2007.

26. Gilad AA, McMahon MT, Walczak P, Winnard PT Jr, Raman V, van Laarhoven HW, Skoglund CM, Bulte JW and van Zijl PC: Artificial reporter gene providing MRI contrast based on proton exchange. Nat Biotechnol 25: 217-219, 2007. 